ITC 4/46

Journal of Information Technology and Control

Vol. 46 / No. 4/ 2017

pp. 470-483

DOI 10.5755/j01.itc.46.4.14163

(c) Kaunas University of Technology

\section{An Electronic Public Engineering Project Bidding}

Protocol via a Subliminal Channel
Accepted after revision 2017/10/23

\title{
An Electronic Public Engineering Project Bidding Protocol via a Subliminal Channel
}

\section{Chin-Ling Chen}

Department of Computer Science and Information Engineering, Chaoyang University of Technology, Taichung 41349, Taiwan, (R.O.C.), e-mail: clc@mail.cyut.edu.tw

School of Information Engineering, Changchun Sci-Tech University, 1699 Donghua Street, Shuangyang District, Changchun City, Jilin Province, 130600, China, e-mail: wkhyoyo@163.com

\section{Kun-Hao Wang}

School of Information Engineering, Changchun Sci-Tech University, 1699 Donghua Street, Shuangyang District, Changchun City, Jilin Province, 130600, China, e-mail: wkhyoyo@163.com

\section{Woei-Jiunn Tsaur}

Computer Center, National Taipei University, Taiwan, R.O.C., e-mail: wjtsaur@mail.ntpu.edu.tw

\section{Jung-Hsuan Chen}

Department of Industrial Education, National Taiwan Normal University, Taipei, 10610 Taiwan, R.O.C., e-mail: jhchen@ntnu.edu.tw

\section{Chien-Hung Chen}

Department of Computer Science and Information Engineering, Chaoyang University of Technology, Taichung 41349, Taiwan, (R.O.C.), e-mail: bitz823@hotmail.com 
Due to the rapid development of the Internet, many Internet applications have recently become very widely used. Internet security has therefore become an important issue. This paper proposes an electronic public engineering project bidding protocol via a subliminal channel. In the proposed scheme, the subliminal channel can protect a bidder's interests, while allowing an official agent to make a fair arbitration. The proposed scheme is non-repudiable, untraceable and offers fair arbitration of public engineering projects, but is also resistant to replay, forgery and insider attacks, thus enhancing both security and fairness.

KEYWORDS: Subliminal channel, Fair arbitration, Security, Non-repudiation, Digital signature.

\section{Introduction}

In recent years, the Internet has developed rapidly, and many Internet transaction applications have become popular. For example, many public construction engineering projects now conform to public, fair and efficient requirements via Internet technology. For example, in e-bidding cases, some important issues should be of concern. The bidding price should be protected to defend against insider attack, and there should be a fair arbitration mechanism to arbitrate accusations.

In 1983, Simmons [15-17] first proposed the subliminal channel mechanism. Subliminal channels are employed for secret communication; they can be used to deliver subliminal messages between sender and receiver. Subliminal messages cannot be accessed except through the specific receiver. In 1997, Harn and Gong [5] proposed a digital signature using a subliminal channel; they showed how to construct a digital signature scheme with a broadband subliminal channel that does not require a subliminal receiver to share the transmitter's secret signing key. In 2010, Lin et al. [9] proposed a digital signature with multiple subliminal channels, and its applications. The proposed scheme has the advantage that the subliminal receivers cannot forge a valid signature since they do not share the signer's secret key. It can also provide more than one independent subliminal message.

In 1998, Subramanian [18] presented the design and verification of a secure electronic auction protocol. This protocol ensured anonymity, security, privacy, atomicity and low overhead. Hwang et al. [6] proposed adding timestamps to the secure electronic auction protocol in 2002. They found the drawback of Subramanian's protocol and proposed an electronic auction protocol with improved robustness. In 2006, Liaw et al. [8] proposed an electronic online bidding auction protocol with both improved security and efficiency. This protocol not only satisfies the requirements for the electronic auction properties of anonymity, security, privacy, atomicity and low overhead cost, but also adds the properties of non-repudiation, untraceability, auditablity, one-time registration and unlinkability. In 2008, Chun et al. [3] proposed a bidder-anonymous English auction scheme with privacy and public verifiability. The proposed scheme provided the following security features: anonymity, traceability, no framing, unforgeability, non-repudiation, fairness, public verifiability, unlinkability among various auction rounds, linkability within a single auction round, bidding efficiency, one-time registration, and easy revocation. In 2012, Xiong et al. [19] proposed a bidder-anonymous English auction protocol based on revocable ring signature. The proposed protocol has three appealing characteristics: first, it offers conditional privacy-preservation: while the auctioneer can verify that a bidder is an authorized participant in the system, only the collaboration of auctioneer and registration manager can reveal the true identity of a malicious bidder. Second, it is a one-time registration: the bidder can take part in plural auctions with one time registration. Third, it is spontaneous: the bidder can bid without interaction with the auctioneer and other bidders. Fan et al. [4] proposed a multi-recastable e-bidding game with dual-blindness. The proposed protocol allows all participants to take part in a sequence of different auctions for various products unlimitedly after performing a onetime registration, where the winner does not need to re-register either. They formally proved the security of the proposed scheme and also provided comparisons to show that it was the most efficient one, compared with previous works. In order to defend against known attacks, some applications should embed authentication mechanisms [10-11] to ensure that security requirements can also be guaranteed. 
This paper proposes a novel scheme for a fair bidding transaction for public construction engineering projects. The fair bidding transactions need to protect the bidder's privacy and uphold a fair transaction process. The proposed scheme is not only able to protect the bidder's privacy, but can also support fair arbitration via a subliminal channel.

The proposed scheme should have the following characteristics:

1 Non-repudiation [2]: Non-repudiation refers to the ability to ensure that parties cannot deny the authenticity of their signature on a message which they have sent.

2 Fair arbitration [1]: The fair arbitration mechanism can allow participating parties access to fair arbitration when they have doubts regarding aspects of the project.

3 Blind message [14]: The blind message mechanism can be provided to protect the bidder's privacy, and other persons cannot extract the message, aside from the owner of the blind factor.

4 Unlinkability [4]: No one can trace a specific bidder from the transaction message.

5 One-time registration [4]: The protocol only requires one-time registration for each bidder.

6 Auditability [4]: Auditability is a third-party application which can assist an auctioneer to find the real identity of a bidder in certain cases, or if disputes occur.

7 Defence against replay attack [1]: A replay attack occurs when an attacker copies the message between two parties and replays it to one or more parties in order to gain access to sensitive information.

8 Defence against forgery attack [14]: In a forgery attack, an attacker masquerading as a legal party transmits the message to obtain the other party's trust, and thereby gains access to sensitive information.

9 Defence against insider attack [14]: The attacker can access sensitive information using a legal identity to achieve an insider attack.

The remainder of this paper is arranged as follows. Section 2 introduces the framework of the proposed scheme. Section 3 offers a security analysis of the proposed scheme. Section 4 discusses the computation costs of the proposed scheme, and makes a security comparison with related works. Finally, conclusions regarding the proposed scheme are drawn in Section 5.

\section{The Proposed Scheme}

The overview of the proposed scheme is shownin Figure 1. There are five parties involved in the scheme:

1 Bidder (BI): A person or a company that wants to take part in a bidding case

2 Public Construction Commission (PCC): The organization which has a private enterprise or government entity hold a bidding operation

3 Bank (BK): The bank of the Bidder and Public Construction Commission

4 Proxy Server (PS): A trusted proxy server used to transfer and store important information

5 Official Agent (OA): A trusted and fair arbitrator

Step 1: $B I \rightarrow B K, P S \& P C C \rightarrow B K, P S: B I$ and $P C C$ register with the BK and PS, respectively.

Step 2: BI $\rightarrow$ BK: When a bidder wants to take part in the bid, the bidder sends the bidding bond and related information to the bank.

Step 3: BK $\rightarrow$ BI: After receiving the message, BK stores the bidding bond, signs the response message and sends it to the BI.

Step 4: BI $\rightarrow$ PCC: The BI makes the bidding message and uses the blind factor to make a blind signature. It then sends the blind message to the PCC.

Step 5: PCC $\rightarrow$ BI: After receiving the blind message, the PCC will sign the message and send it back to the BI.

Step 6: BI $\rightarrow$ PS: The BI makes the bidding message, which includes the blind factor and the bidder's identity. After that, the BI sends the bidding message to the PS.

Step 7: PS $\rightarrow$ BI: The PS sends the response message to the BI.

Step 8: PS $\rightarrow$ PCC: The PS sends the blind factor and bidding identity to the PCC.

Step 9: PCC $\rightarrow$ PS: the PCC sends the response message to the PS.

Step 10: PCC $\rightarrow$ BI: The PCC publishes the winner.

Step 11: BI $\rightarrow$ OA or PCC $\rightarrow$ OA: When the BI or PCC doubts this bidding case, they can send the blind message, blind factor and subliminal message to the OA to request arbitration. 


\section{Figure 1}

The overview of our scheme

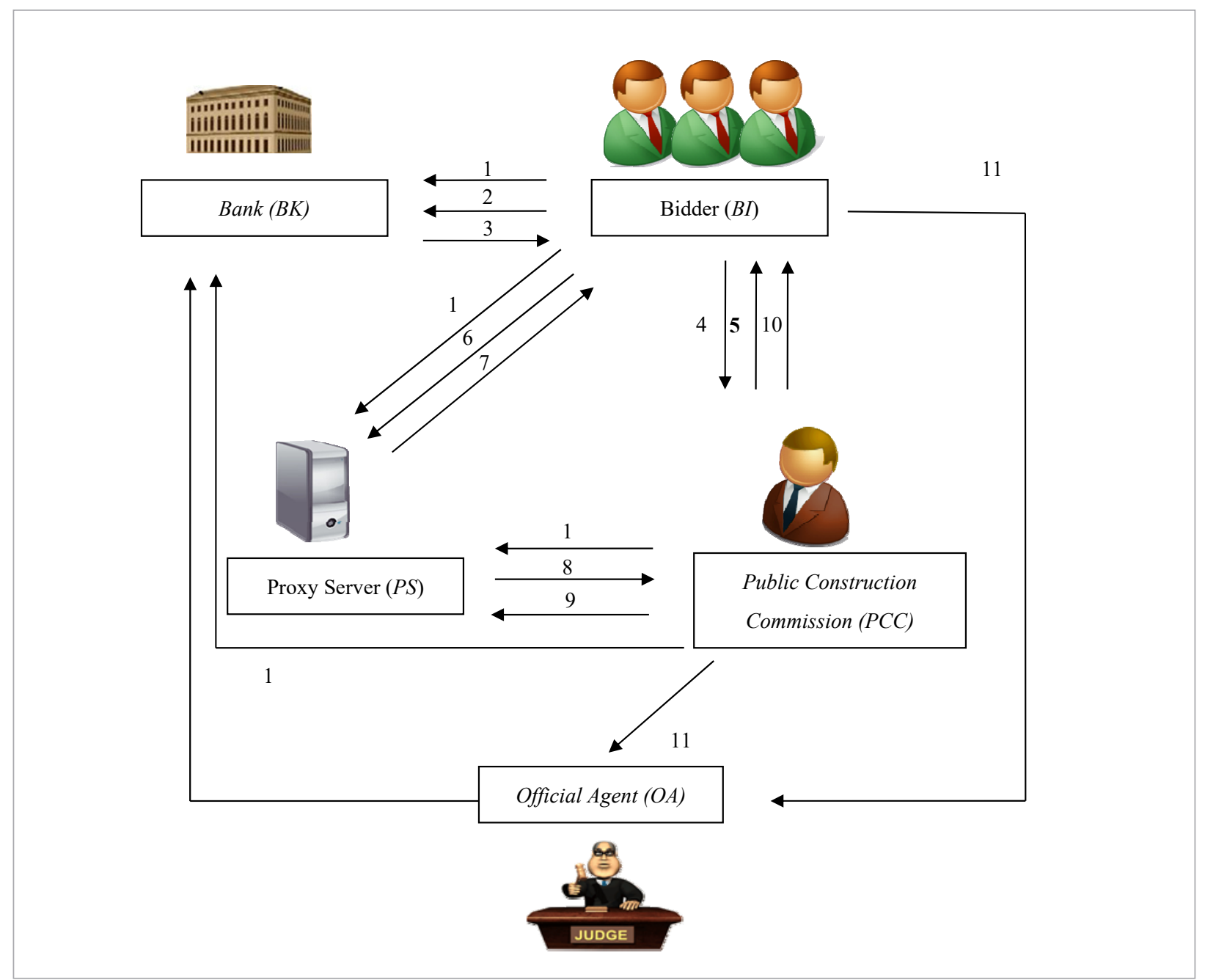

The following notations are used in the proposed protocol:

$x_{B I}, x_{B K}$,
$x_{P C C}, x_{P S}$,

$x_{O A}$ respectively

$y_{B I}, y_{B K}$, : the BI, BK, PCC, PS and OA's public keys, $y_{P C C}, y_{P S}$, respectively, for example: $y_{B I}=g^{x_{B I}} \bmod p$, yOA where $\mathrm{g}$ is a randomly chosen generator of the multiplicative group Zp

$\left(d_{i}, s_{i}\right) \quad$ : the $i$ th signature pair

$T_{X-Y} \quad:$ the timestamp generated by $\mathrm{X}$, and trans-

$\begin{array}{ll} & \text { ferred to } \mathrm{Y} \\ M_{s} & \text { : the subliminal message }\end{array}$
$M X-Y \quad$ : the message is transferred from $\mathrm{X}$ to $\mathrm{Y}$

$C_{i} \quad$ : the $i$ th cipher message

$h() \quad$ :a one-way hash function

$A ? \stackrel{B}{=} \quad$ : determine if $\mathrm{A}$ is equal to $\mathrm{B}$.

\subsection{The Registration Phase}

In the proposed scheme, the encryption and decryption mechanism is used to protect messages, based on the ElGamal scheme. The BI and PCC need to register with the bank $(B K)$ and proxy server $(P S)$. Then, the bank and proxy server store the BI and PCC's identities: $I D_{B I}$ and $I D_{P C C}$, respectively. After this, the participating parties receive their private and public keys. 
Therefore, the participating parties can select their own private keys: $x_{B I}, x_{B K}, x_{P C C}, x_{P S}$ and $x_{O A}$. It can then compute the responding public key.

Step 1: The BI, BK, PCC, PS and OA choose their private keys $x_{B I}, x_{B K}, x_{P C C}, x_{P S}$ and $x_{O A}$, respectively. Then these parties compute public keys $y_{B I}, y_{B K}, y_{P C C}, y_{P S}$ and $y_{O A}$, respectively, as follows:

$y_{B I}=g^{x_{B I}} \bmod p$
$y_{B K}=g^{x_{B K}} \bmod p$
$y_{P C C}=g^{x_{P C C}} \bmod p$
$y_{P S}=g^{x_{P S}} \bmod p$
$y_{O A}=g^{x_{O A}} \bmod p$

\subsection{The Bidding Phase}

The BI sends the bidding bonds to the bank (BK), and then the $\mathrm{BK}$ returns the response message to the BI. After this, the BI sends the blind message to the PCC to sign it, and the PCC returns the blind message. The $\mathrm{BI}$ then sends the bidding message to the PS. The bidding phase is shown in Figure 2.

Step 1: The BI computes the bidding bonds message $M_{B I-B K}$ :

$M_{B I-B K}=\left(A C C_{B I}, A C C_{P C C}, I D_{p r o}, A M O U N T, I D_{B I}, T_{B I-B K}\right)$.

The BI then chooses a random number $r_{1}$ and computes the ciphertexts $C_{1}$ and $C_{2}$ as follows:

$$
C_{1}=g^{r_{1}} \bmod p
$$

$C_{2}=M_{B I-B K} \times y_{B K}{ }^{{ }_{1}} \bmod p$.

The BI then sends the ciphertexts $\left(C_{1}, C_{2}\right)$ to the BK.

Step 2: Upon receiving the ciphertexts $\left(C_{1}, C_{2}\right)$, the BK uses the private key $x_{B K}$ to decrypt the message:

$$
\begin{aligned}
& w=\left(C_{1}^{x_{B K}}\right)^{-1} \bmod p \\
& M_{B I-B K}=C_{2} \times w \bmod p \\
& M_{B K-B I}=\left(A C C_{B I}, A C C_{P C C}, M_{t r a}, I D_{p r o}, T_{B K-B I}\right) .
\end{aligned}
$$

The BK then chooses a random number $r_{2}$, makes a response message $M_{B K-B I}$ and computes a signature $\left(d_{1}, s_{1}\right)$ :

$d_{1}=g^{r_{2}} \bmod p$

$s_{1}=r_{2}^{-1}\left(M_{B K-B I}-x_{B K} d_{1}\right) \bmod p-1$.

After this, the BK sends the signature $\left(d_{1}, s_{1}\right)$ and message $M_{B K-B I}$ to the BI.

Step 3: After receiving the signature $\left(d_{1}, s_{1}\right)$ and message $M_{B K-B I}$, the BI uses the public key $y_{B K}$ to verify the signature:

$y_{B K}{ }^{d_{1}} \times d_{1}^{S_{1}} \stackrel{?}{=} g^{M_{B K-B I}} \bmod p$.

Then, the BI creates a subliminal message $M_{s}$ and message $M_{B I-P C C}$ as follows:

$M_{s}=\left(\left(d_{1}, s_{1}\right), x_{B I}, I D_{B I}, I D_{P C C}, M_{\mathrm{inf}}, I D_{p r o}\right)$

$M_{B I-P C C}=I D_{p r o}{ }^{M s} \bmod p-1$.

It sends a blind signature request message $M_{r e q}$ to the PCC.

Step 4: The PCC chooses a random number $k$ and computes parameter $\tilde{d}_{2}$ :

$\tilde{d}_{2}=g^{k} \bmod p$.

The PCC sends the parameter $\tilde{d}_{2}$ to the BI.

Step 5: The BI chooses random numbers $(a, b, c)$ and computes:

$d_{2}=\tilde{d}_{2}{ }^{a} y^{b} g^{c} \bmod p$

$\tilde{M}_{B I-P C C}=a^{-1}\left(d_{2}+h\left(M_{B I-P C C}\right)-b\right)-\tilde{d}_{2} \bmod p-1$.

$M_{B I-P C C}$ is then sent to the PCC to sign the message.

Step 6: The PCC computes:

$$
\tilde{s}_{2}=\left(\tilde{d}_{2}+\tilde{M}_{B I-P C C}\right) x_{P C C}-k \bmod p-1
$$

and then sends $\tilde{S}_{2}$ to the BI.

Step 7: After receiving the blind signature $\tilde{s}_{2}$, the BI decrypts the blind message and obtains the signature:

$s_{2}=a \tilde{s_{2}}-c \bmod p-1$ 
Figure 2

The scenario of the bidding phase

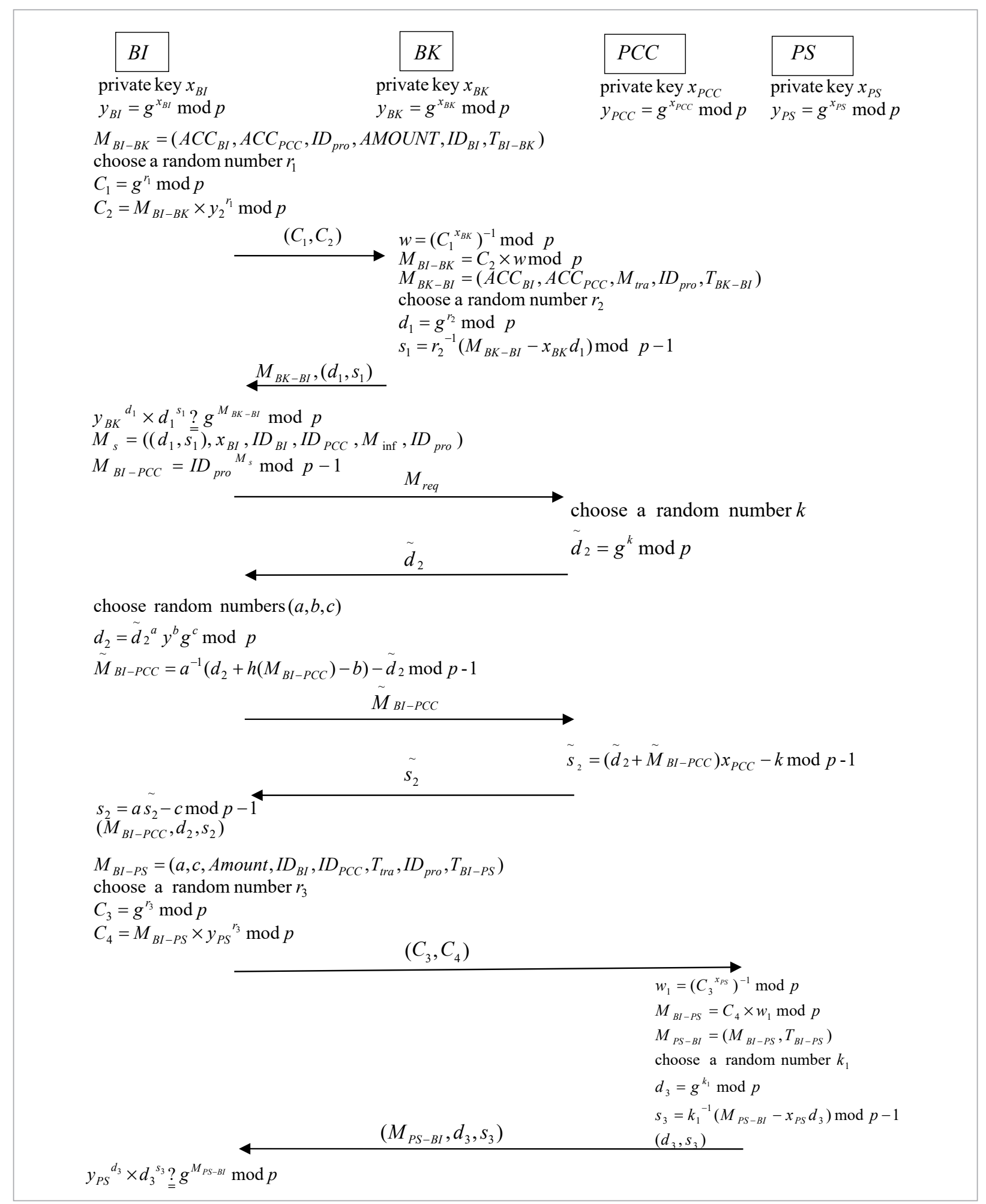


so that the BI can obtain the signature $\left(M_{B I-P C C}, d_{2}, s_{2}\right)$. The BI makes a bidding message for the PS:

$M_{B I-P S}=\left(a, c, A M O U N T, I D_{B I}, I D_{P C C}, T_{t r a}, I D_{p r o}, T_{B I-P S}\right) \cdot$ (22)

It chooses a random number $r_{3}$ and computes ciphertexts $\left(C_{3}, C_{4}\right)$ :

$C_{3}=g^{r_{3}} \bmod p$

$C_{4}=M_{B I-P S} \times y_{P S}^{r_{3}} \bmod p$

It sends the ciphertexts $\left(C_{3}, C_{4}\right)$ to the PS.

Step 8: After receiving the ciphertext $\left(C_{3}, C_{4}\right)$, the PS uses the private key $x_{P S}$ to decrypt the messages $C_{3}$ and $C_{4}$ :

$$
w_{1}=\left(C_{3}^{x_{P S}}\right)^{-1} \bmod p
$$

$M_{B I-P S}=C_{4} \times w_{1} \bmod p$.

Then, the BK chooses a random number $k_{1}$ and sends a response message $M_{P S-B I}$ (where $M_{P S-B I}=\left(M_{B I-P S}\right.$, $\left.T_{B I-P S}\right)$ ), chooses a random number $k_{1}$ and computes a signature $\left(d_{3}, s_{3}\right)$ : $d_{3}=g^{k_{1}} \bmod p$

$s_{3}=k_{1}^{-1}\left(M_{P S-B I}-x_{P S} d_{3}\right) \bmod p-1$.

After this, the PS sends the signature $\left(d_{3}, s_{3}\right)$ and message $M_{P S-B I}$ to the BI.

Step 9: Upon receiving the signature $\left(d_{3}, s_{3}\right)$ and message $M_{P S-B I}$, the BI verifies the signature as follows:

$y_{P S}{ }^{d_{3}} \times d_{3} \stackrel{s_{3}}{\stackrel{?}{=}} g^{M_{P S-B I}} \bmod p$.

\subsection{The Bidding Phase}

After the deadline of the casting bid, PS sends the blind factor to the PCC. The PCC then uses the blind factor to obtain the bidding price. After this, the PCC publishes the winner. The scenario of the opening bid phase is shown in Figure 3.

Step 1: The PS chooses a random number $k_{2}$, and creates a message $M_{P S V}$ (where $M_{P S V}=\left(M_{B I-P S}, T_{P S-P C C}\right)$ ) and a signature $\left(d_{4}, s_{4}\right)$ :

$d_{4}=g^{k_{2}} \bmod p$

\section{Figure 3}

The scenario of the opening bid phase

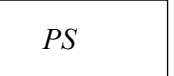

choose a random number $k_{2}$

$M_{P S V}=\left(M_{B I-P S}, T_{P S-P C C}\right)$

$d_{4}=g^{k_{2}} \bmod p$

$s_{4}=k_{2}^{-1}\left(M_{P S V}-x_{P S} d_{4}\right) \bmod p-1$

$\left(d_{4}, s_{4}\right)$

$M_{P S-P C C}=\left(a, c, I D_{B I}, I D_{P C C}\right.$, Amount, $\left.I D_{p r o}, T_{P S-P C C}, d_{4}, s_{4}, M_{P S V}\right)$

choose a random number $r_{4}$

$C_{5}=g^{r_{4}} \bmod p$

$C_{6}=M_{P S-P C C} \times y_{P C C}{ }^{r_{4}} \bmod p$

$w_{2}=\left(C_{5}^{x_{P C C}}\right)^{-1} \bmod p$

$M_{P S-P C C}=C_{6} \times w_{2} \bmod p$

$\left(C_{5}, C_{6}\right)$

$y_{P S}{ }^{d_{4}} \times d_{4}{ }^{s_{4}} \stackrel{?}{=} g^{M_{P S V}} \bmod p$

$\left.M_{P C C-P S}=\overline{\overline{(}} M_{P C C-R E S}, T_{P C C-P S}\right)$

choose a random number $k_{3}$

$d_{5}=g^{k_{3}} \bmod p$

$s_{5}=k_{3}^{-1}\left(M_{P C C-P S}-x_{P C C} d_{5}\right) \bmod p-1$

$\left(d_{5}, s_{5}\right), M_{P C C-P S}$

$y_{P C C} d_{5} \times d_{5} \stackrel{s_{5}}{=} g^{M_{P C C-P S}} \bmod p$

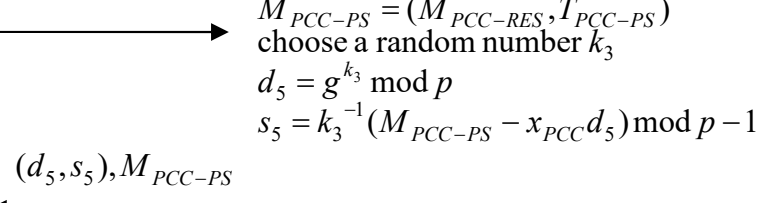


$s_{4}=k_{2}^{-1}\left(M_{P S V}-x_{P C} d_{4}\right) \bmod p-1$.

The PS then creates a bidding message:

$$
\begin{aligned}
& M_{P S-P C C}=\left(a, c, I D_{B I}, I D_{P C C},\right. \text { Amount } \\
& \left.I D_{\text {pro }}, T_{P S-P C C}, d_{4}, s_{4}, M_{P S V}\right) .
\end{aligned}
$$

Next it chooses a random number $r_{4}$ and computes ciphertexts $\left(C_{5}, C_{6}\right)$ :

$C_{5}=g^{r_{4}} \bmod p$
$C_{6}=M_{P S-P C C} \times y_{P C C}^{r_{4}} \bmod p$

It then sends the ciphertexts $\left(C_{5}, C_{6}\right)$ to the PCC.

Step 2: After receiving the ciphertexts $\left(C_{5}, C_{6}\right)$, the PCC uses the private key $x_{P C C}$ to decrypt the message:
$w_{2}=\left(C_{5}^{x_{P C C}}\right)^{-1} \bmod p$
$M_{P S-P C C}=C_{6} \times w_{2} \bmod p$.

The PCC verifies if the bidding message is valid or not:

$y_{P S}{ }^{d_{4}} \times d_{4} \stackrel{s_{4}}{=} g^{M_{P S V}} \bmod p$.

Then, the PCC generates a response message $M_{P C C-P S}$ (where $M_{P C C-P S}=\left(M_{P C C-R E S}, T_{P C C-P S}\right)$ ).

The PCC chooses a random number $k_{3}$ and computes the signature $\left(d_{5}, s_{5}\right)$ as follows:

$d_{5}=g^{k_{3}} \bmod p$
$s_{5}=k_{3}^{-1}\left(M_{P C C-P S}-x_{P C C} d_{5}\right) \bmod p-1$

Afterwards, the PCC sends the signature $\left(d_{5}, s_{5}\right)$ and $M_{P C C-P S}$ to the PS.

Step 3: Upon receiving the signature $\left(d_{5}, s_{5}\right)$, the PS verifies the signature:

$$
y_{P C C}{ }^{d_{5}} \times d_{5} \stackrel{s_{5}}{=} g^{M_{P C C-P S}} \bmod p
$$

\subsection{The Official Agent Arbitration Phase}

In this phase, if there are concerns regarding the $\mathrm{BI}$ or PCC, the OA can offer fair arbitration by blind message and subliminal message. Once the accusation holds, the winner can request the bank to reveal the information of the accused party.

Case 1: The BI takes the subliminal message, blind message and blind factor to the OA. The OA then uses the related information to make a fair arbitration. The overview of the arbitration phase (PCC is accused) is shown in Figure 4:

Step 1: The BI generates an accusation message $M_{B I-O A}$ :

$$
\begin{aligned}
& M_{B I-O A}=\left(M_{S}, I D_{B I}, I D_{P C C}, I D_{\text {pro }},\right. \\
& \left.M_{B I-P C C}, M_{P S V}, d_{2}, s_{2}\right) .
\end{aligned}
$$

It then chooses a random number $r_{5}$ and computes the ciphertexts $\left(C_{r}, C_{8}\right)$ :

$$
\begin{aligned}
& C_{7}=g^{r_{5}} \bmod p \\
& C_{8}=M_{B I-O A} \times y_{O A}^{r_{5}} \bmod p .
\end{aligned}
$$

It then sends the ciphertexts $\left(C_{7}, C_{8}\right)$ to the OA.

Step 2: Upon receiving the ciphertexts $\left(C_{7}, C_{8}\right)$, the OA uses the private key $x_{O A}$ to decrypt the message:

$$
\begin{aligned}
& w_{3}=\left(C_{7}^{x_{O A}}\right)^{-1} \bmod p \\
& M_{B I-O A}=C_{8} \times w_{3} \bmod p .
\end{aligned}
$$

The OA then verifies the signature $\left(d_{2}, s_{2}\right)$ :

$$
y_{P C C} d_{2}+h\left(M_{B I-P C C}\right) \stackrel{?}{=} d_{2} g^{s_{2}} \bmod p
$$

If Equation (4r) holds, it then verifies the message $M_{B I-P C C}$ :

$M_{B I-P C C} \stackrel{?}{=} I D_{\text {pro }}^{M_{S}} \bmod p-1$.

Using subliminal message $M_{S}$ and accusation message $M_{B I-O A}$, the OA is able to make a fair arbitration.

Case 2: The PCC takes the blind message and blind factor to the OA. Then, the OA uses the related information to make a fair arbitration. The overview of the arbitration phase (BI is accused) is shown in Figure 5:

Step 1: The PCC generates an accusation message $M_{P C C-O A}$ (where $M_{P C C-O A}=\left(I D_{B I}, I D_{P C C}, I D_{p r o}, M_{P S-P C C}\right.$, $\left.\left.D_{P S}, d_{4}, s_{4}\right)\right)$.

The PCC then chooses a random number $r_{6}$ and computes ciphertexts $\left(C_{9}, C_{10}\right)$ : 
Figure 4

The overview of the arbitration phase (PCC is accused)

$$
\begin{aligned}
& \begin{array}{|l|l|}
\hline B I & \\
\hline
\end{array} \\
& M_{B I-O A}=\left(M_{S}, I D_{B I}, I D_{P C C}, I D_{p r o}, M_{B I-P C C}, M_{P S V}, d_{2}, s_{2}\right) \\
& \text { choose a random number } r_{5} \\
& C_{7}=g^{r_{5}} \bmod p \\
& C_{8}=M_{B I-O A} \times y_{O A}^{r_{5}} \bmod p \quad\left(C_{7}, C_{8}\right) \\
& w_{3}=\left(C_{7}^{x_{O A}}\right)^{-1} \bmod p \\
& M_{B I-O A}=C_{8} \times w_{3} \bmod p \\
& y_{P C C} d_{2}+h\left(M_{B I-P C C}\right) \stackrel{?}{\underline{\bar{s}}} d_{2} g^{s_{2}} \bmod p \\
& M_{B I-P C C} \stackrel{?}{=} I D_{\text {pro }}{ }^{\overline{M_{S}}} \bmod p-1
\end{aligned}
$$

\section{Figure 5}

The overview of the arbitration phase (BI is accused)

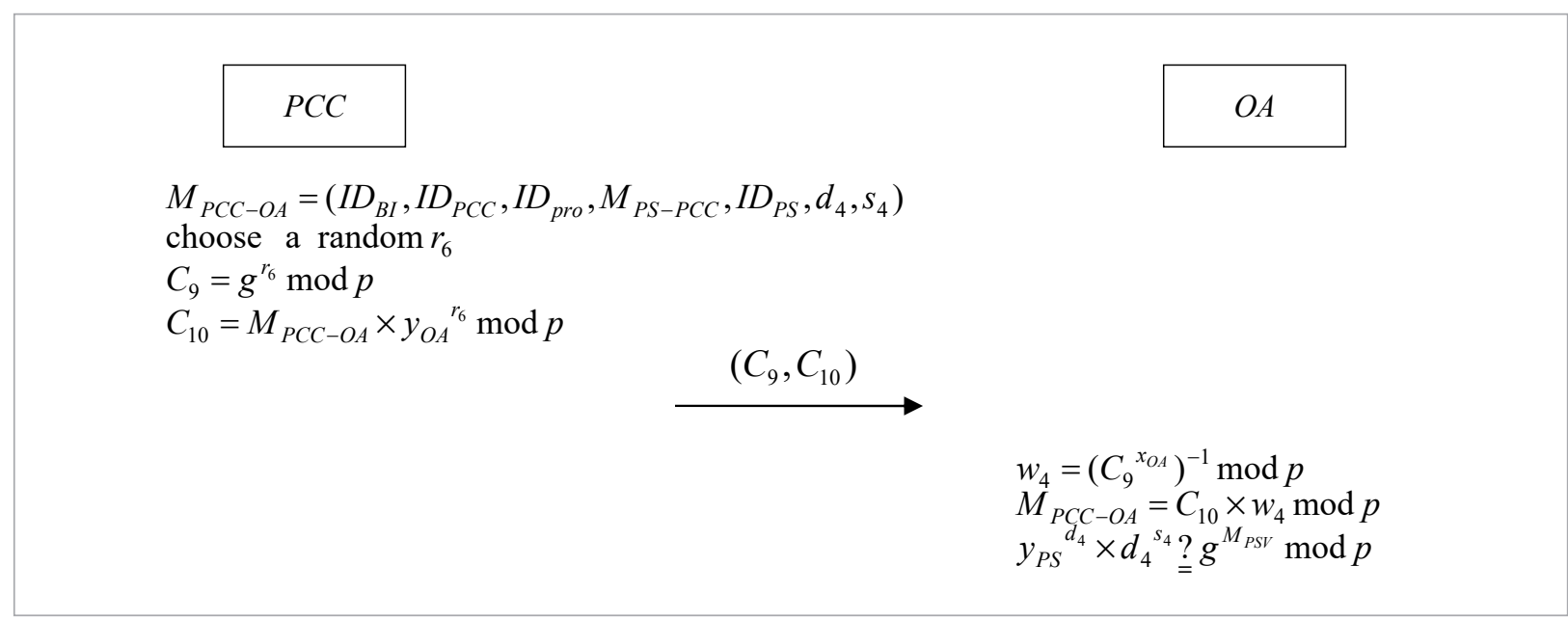

$$
\begin{aligned}
& C_{9}=g^{r_{6}} \bmod p \\
& C_{10}=M_{P C C-O A} \times y_{O A}{ }^{r_{6}} \bmod p .
\end{aligned}
$$

It then sends the ciphertexts $\left(C_{9}, C_{10}\right)$ to the OA.

Step 2: After receiving the ciphertexts $\left(C_{9}, C_{10}\right)$, the OA uses the private key $x_{O A}$ to decrypt the message $M_{P C C-O A}$ :

$$
w_{4}=\left(C_{9}{ }^{x_{O A}}\right)^{-1} \bmod p
$$

$$
M_{P C C-O A}=C_{10} \times w_{4} \bmod p .
$$

The OA then verifies the signature $\left(d_{4}, s_{4}\right)$ :

$$
y_{P S}{ }^{d_{4}} \times d_{4} \stackrel{s_{4}}{=} \stackrel{g^{M}}{M_{P S V}} \bmod p .
$$

Using verified message $M_{P S V}$ and accusation message $M_{P C C-O A}$, the OA can make a fair arbitration. The summary flowchart of the arbitration phase is shown in Figure 6. 
Figure 6

The flowchart of the arbitration phase

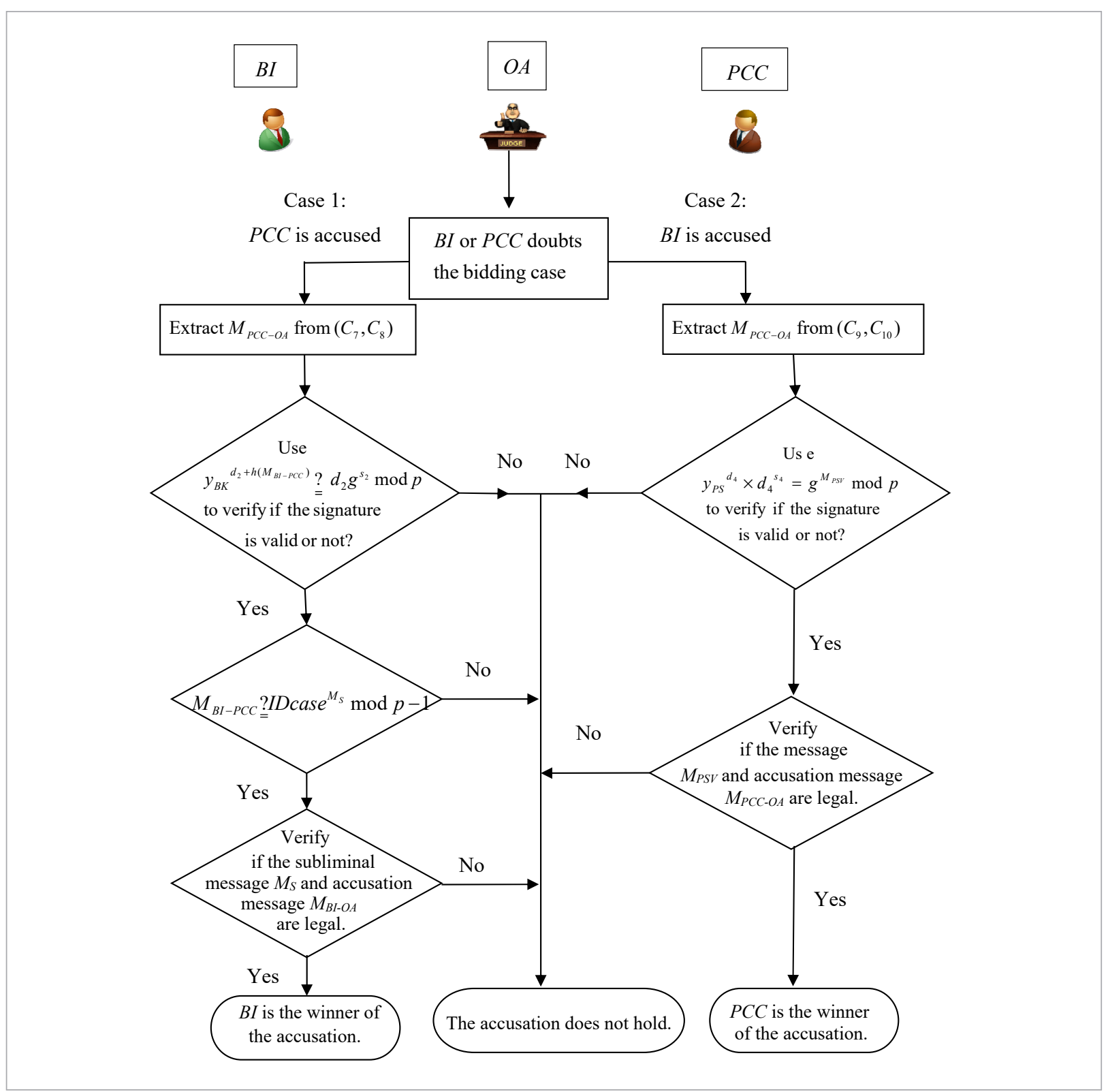

\section{Security Analysis}

\subsection{The Official Agent Arbitration Phase}

The proposed protocol employs a digital signature mechanism to solve the non-repudiation problem with ElGamal signature and blind signature. The verifications are shown in Table 1.

\subsection{Fair Arbitration}

This section will illustrate how the proposed scheme provides fair arbitration.

Case 1: The BI doubts the PCC

In the arbitration phase, after receiving the message $\left(C_{r}, C_{8}\right)$ from the $\mathrm{BI}$, the OA will decrypt the ci- 
Table 1

The non-repudiation issues

\begin{tabular}{|c|c|c|c|}
\hline Non-repudiation proof & Proof issuer & Proof holder & Verification \\
\hline$\left(M_{B K-B I}, d_{1}, s_{1}\right)$ & $\mathrm{BK}$ & BI & $y_{B K}^{d_{1}} \times d_{1}^{S_{1}} \stackrel{?}{=} g^{M_{B K-B I}} \bmod p$ \\
\hline$\left(M_{B I-P C C}, d_{2}, s_{2}\right)$ & BK & BI & $y_{B K}{ }^{d_{2}+h\left(M_{B I-P C C}\right)} \bmod p \stackrel{?}{=} d_{2} g^{s_{2}} \bmod p$ \\
\hline$\left(M_{P S-B I}, d_{3}, s_{3}\right)$ & PS & $\mathrm{BI}$ & $y_{P S}{ }^{d_{3}} \times d_{3} \stackrel{s_{3}}{=} ? g^{M_{P S-B I}} \bmod p$ \\
\hline$\left(M_{P S V}, d_{4}, s_{4}\right)$ & PS & PCC & $y_{P S}{ }^{d_{4}} \times d_{4} \stackrel{s_{4}}{=} \stackrel{g^{M_{P S V}}}{\bmod p}$ \\
\hline$\left(M_{P C C-P S}, d_{5}, s_{5}\right)$ & PCC & PS & $y_{P C C}{ }^{d_{5}} \times d_{5}{ }^{s_{5}} \stackrel{?}{\underline{?}} g^{M_{P C C-P S}} \bmod p$ \\
\hline
\end{tabular}

phertexts and verify the BI's blind message $M_{B I-P C C}$ : $y_{B K}{ }^{d_{2}+h\left(M_{B l-P C C}\right)} \bmod p \stackrel{?}{=} d_{2} g^{s_{2}} \bmod p$. If the equation holds, the OA will verify the reality of message $M_{B I-P C C}: M_{B I-P C C}$ ? $I D_{\text {case }}{ }^{M_{S}} \bmod p-1$. Then, the OA uses $M_{B I-P C C}$ and $M_{S}$ to arbitrate the accusation.

The derivation of the blind signature verification is shown as follows:

$$
\begin{aligned}
& { }_{P C C}{ }^{d_{2}+h\left(M_{B I-P C C}\right)} \bmod p \\
& =d_{2} g^{s_{2}} \bmod p \\
& =\left(g^{k a} g^{x_{P C C} b} g^{c}\right) g^{a \tilde{s}_{2}-c} \\
& =g^{k a} g^{x_{P C C} b} g^{a s_{2}} \\
& =g^{k a} g^{x_{P C C} b} g^{a\left[\left(\tilde{d}_{2}+\tilde{M}_{B I-P C C} x_{P C C}-k\right]\right.} \\
& =g^{k a} g^{x_{P C C} b} g^{a x_{P C C}} \tilde{d}_{2} g^{a x_{2} \tilde{M}_{B I-P C C}} / g^{k a} \\
& =g^{x_{P C C} b} g^{a x_{P C C}} \tilde{d}_{2} g^{a x_{P C C} \tilde{M}_{B I-P C C}} \\
& =g^{x_{2} b} g^{a x_{2} d_{2}} g^{a x_{2}\left(a^{-1}\left(d_{2}+h\left(M_{B I-P C C}\right)-b\right)-d_{2}\right)} \\
& =g^{x_{P C C} b} g^{a x_{P C C}} \tilde{d}_{2} g^{x_{P C C}\left(d_{2}+h\left(\tilde{M}_{B I-P C C}\right)\right)} / g^{b x_{P C C}} g^{a x_{P C C}} \tilde{d}_{2} \\
& =g^{x_{P C C}\left(d_{2}+h\left(\tilde{M}_{B I-P C C}\right)\right)} \\
& =y_{P C C} d_{2}+h\left(M_{B I-P C C}\right)
\end{aligned}
$$

Case 2: The BI doubts the PCC

In the arbitration phase, after receiving the message $\left(C_{9}, C_{10}\right)$ from the PCC, the OA will decrypt the ciphertexts and send the message $M_{P C C-O A}$. It then verifies the PS's signature $\left(M_{P S V}, d_{4}, s_{4}\right): y_{P S}{ }^{d_{4}} \times d_{4} \stackrel{S_{4}}{=} g^{M_{P S V}} \bmod p$.
If the equation holds, then the $O A$ uses $M_{P S V}$ to arbitrate the accusation. This design can ensure fair arbitration.

The derivation of signature verification is shown as follows:

$$
\begin{aligned}
& g^{M_{P S V}} \bmod p \\
& =y_{P S}{ }^{d_{4}} \times d_{4}^{s_{4}} \bmod p \\
& =g^{x_{P S}{ }^{d_{4}}} \times g^{k_{2}{ }^{k_{2}{ }^{-1}\left(M_{P S V}-x_{P S} d_{4}\right)}} \\
& =g^{x_{P S}{ }^{d_{4}}} \times g^{\left(M_{P S V}-x_{P S} d_{4}\right)} \\
& =g^{M_{P S V}} .
\end{aligned}
$$

\subsection{The Blind and Unlinkable Issue}

In the bidding phase, the BI sends the blind message to the PCC to sign the message. The blind signature scheme is secure against malicious attackers who work in the PCC. Even if the PCC receives the bidding message from the BI, the PCC does not know the message in the bidding phase. In the opening bid phase, the PCC retrieves the blind message in order to use the blind factor and other information from the PS. Therefore, the malicious attacker cannot obtain the BI's bidding message and identity in the bidding phase.

\subsection{Auditability}

In the official agent arbitration phase, the OA can ask the bank for the information of the BI and PCC when it receives an accusation message. Therefore, the proposed protocol provides auditability to help the OA offer fair arbitration. 


\subsection{One-Time Registration}

The BI and PCC only need to register once with the $\mathrm{BK}$ and PS. This reduces computation cost and creates greater convenience for the bidder.

\subsection{Defense Against Known Attacks}

\subsubsection{Replay Attack}

The proposed scheme includes a timestamp mechanism, which varies for each transaction. If a malicious attacker attempts to replay a message, it will fail, making replay attacks impossible.

\subsubsection{Forgery Attack}

In the bidding phase, when the BI sends the bidding message to the PCC, it only sends the blind message; it is very difficult for an attacker to forge the message to pretend to be the bidder or other parties. The proposed scheme uses ElGamal encryption and decryption mechanism in each transaction. For example: the encryptions in the bidding phase are:

$C_{1}=g^{r_{1}} \bmod p, C_{2}=M_{B I-B K} \times y_{2}{ }^{r_{1}} \bmod p$,

$C_{3}=g^{r_{3}} \bmod p, C_{4}=M_{B I-P S} \times y_{P S}{ }^{r_{3}} \bmod p$

Table 2

The computation cost of our scheme

\begin{tabular}{|c|c|c|c|c|c|}
\hline Phase & $\begin{array}{l}\text { Hwang et al.'s } \\
\text { scheme [6] }\end{array}$ & $\begin{array}{l}\text { Liaw et al.'s } \\
\text { scheme [8] }\end{array}$ & $\begin{array}{l}\text { Chung et al.'s } \\
\text { scheme [3] }\end{array}$ & $\begin{array}{l}\text { Xiong et al.'s } \\
\text { scheme [19] }\end{array}$ & Our scheme \\
\hline The registration phase & $5 T_{E x p}$ & $6 T_{E x p}$ & $5 T_{E x p}+2 T_{M u t}+2 T_{H}$ & $4 T_{E x p}+1 T_{M u t}+2 T_{H}$ & NA \\
\hline The bidding phase & $12 T_{E x p}$ & $10 T_{E x p}$ & $8 T_{E x p}+7 T_{M u t}+6 T_{H}$ & $2 T_{p a i r}+2 T_{E x p}+2 n T_{s c a}$ & $\begin{array}{c}2 T_{H}+18 T_{E x p}+ \\
20 T_{X O R}+20 T_{M u t}\end{array}$ \\
\hline The opening bid phase & NA & NA & $3 T_{E x p}+1 T_{M u t}+1 T_{H}$ & $1 T_{\text {pair }}+2 T_{E x p}+2 n T_{\text {sca }}$ & $13 T_{E x p}+11 T_{X O R}+9 T_{M u t}$ \\
\hline $\begin{array}{l}\text { The product exchange } \\
\text { and the payment phase }\end{array}$ & $10 T_{E x p}$ & $8 T_{E x p}$ & NA & NA & NA \\
\hline $\begin{array}{l}\text { The official agent } \\
\text { arbitration phase }\end{array}$ & NA & NA & NA & NA & $\begin{array}{c}13 T_{E_{\text {Exp }}+13 T_{X O R^{+}}} 10 T_{M u t} \\
\end{array}$ \\
\hline Total & $27 \mathrm{n} T_{E x p}$ & $24 \mathrm{n} T_{E x p}$ & $\begin{array}{c}\left(11 T_{E x p}+8 T_{M u t}+\right. \\
\left.7 T_{H}\right) \mathrm{n}+6 T_{E x p}+ \\
2 T_{M u t}+2 T_{H}\end{array}$ & $\begin{array}{c}\left(3 T_{\text {pair }}+4 T_{E x p}+\right. \\
\left.4 n T_{s c a}\right) \mathrm{n}+4 T_{s c a}^{+} \\
1 T_{M u t}+2 T_{H}\end{array}$ & $\begin{array}{c}44 T_{E x p}+39 T_{M u t}+ \\
44 T_{X O R}+2 T_{H}\end{array}$ \\
\hline Execution time & $\approx 16.2 \mathrm{n} \mathrm{ms}$ & $\approx 14.88 \mathrm{n} \mathrm{ms}$ & $\approx(6.84 \mathrm{n}+3.73) \mathrm{ms}$ & $\approx(11.78+0.3 \mathrm{n}) \mathrm{n} \mathrm{ms}$ & $\approx 27.38 \mathrm{n} \mathrm{ms}$ \\
\hline
\end{tabular}

Note: $\mathrm{n}$ is the number of bidders; $T_{H}$ is the time complexity of one-way hash function; $T_{E x p}$ is the time for executing the modular exponential operation; $T_{M u t}$ is the time complexity for executing the modular multiplication; $T_{X O R}$ is the time for exclusion or operation; $T_{\text {sca }}$ : the time cost of a scalar multiplication; and $T_{\text {pair }}$ : the time cost of a pairing operation.

$T_{\text {pair }}=3.10 \mathrm{~ms}, T_{E x p}=0.62 \mathrm{~ms}$ (on $3 \mathrm{GHz}$ Pentium IV [13]). $T_{\text {pair }} \approx 5 T_{E x p}, T_{\text {sca }} \approx 29 T_{\text {Mut }}, T_{E x p} \approx 240 T_{\text {Mut }}[$ [7,12,20].
And the decryptions are:

$w=\left(C_{1}^{x_{B K}}\right)^{-1} \bmod p$,

$M_{B I-B K}=C_{2} \times w \bmod p$,

$w_{1}=\left(C_{3}^{x_{P S}}\right)^{-1} \bmod p$ and

$M_{B I-P S}=C_{4} \times w_{1} \bmod p$.

Thus, only the real receiver has his/her own private key, and can decrypt the message. Thus attackers cannot achieve forgery attacks.

\subsubsection{Insider Attack}

If an attacker works in the $\mathrm{PCC}$ or has a relationship with PCC staff, he/she will only be able to obtain the deadline of the bid in the bidding phase from these sources, not the bidding price; the proxy server transfers the bidding message to the PCC and uses the blind signature $\left(d_{2}, s_{2}\right)$ to protect the bidding price $M_{\text {inf }}$ in the bidding phase.

\section{Discussion}

This paper compares the computation cost in Table 2, and makes a security comparison in Table 3 . In the

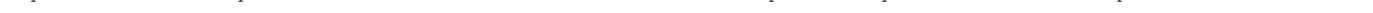




\section{Table 3}

The security comparison of the related works and our scheme

\begin{tabular}{c|c|c|c|c|c}
\hline Security Issues & $\begin{array}{c}\text { Hwang et al.'s } \\
\text { scheme [6] }\end{array}$ & $\begin{array}{c}\text { Liaw et al.'s } \\
\text { scheme [8] }\end{array}$ & $\begin{array}{c}\text { Chung et al.'s } \\
\text { scheme [3] }\end{array}$ & $\begin{array}{c}\text { Xiong et al.'s } \\
\text { scheme [19] }\end{array}$ & $\begin{array}{c}\text { Our } \\
\text { scheme }\end{array}$ \\
\hline Privacy & No & No & Yes & Yes & Yes \\
\hline Atomicity & Yes & Yes & Yes & Yes & Yes \\
\hline Non-repudiation & Yes & Yes & Yes & Yes & Yes \\
\hline Unforgeability & Yes & Yes & Yes & No & Yes \\
\hline Unlinkability & No & No & No & Yes & Yes \\
\hline One-time registration & No & No & Yes & Yes & Yes \\
\hline Auditability & No & Yes & Yes & No & Yes \\
\hline Off-line TTP & No & No & No & & Yes \\
\hline Fair arbitration protocol & No & & No & & Yes \\
\hline
\end{tabular}

proposed scheme, an exponential operation is used to achieve a secure protocol. Although the proposed scheme requires greater computation cost, it is more secure than related works.

\section{Conclusions}

This paper proposes an electronic public engineering project bidding protocol via a subliminal channel which is suitable for the bidding protocol for public construction projects. The proposed scheme satisfies the following properties: non-repudiation, fair arbitration, blind message, unlinkable, one-time registration and auditability. An exponential operation and

\section{References}

1. Chen, C. L., Liao, J. J. A Fair Online Payment System for Digital Content via Subliminal Channel. Electronic Commerce Research and Applications, 2011, 10(3), 279-287. https://doi.org/10.1016/j.elerap.2010.09.001

2. Chen, C. L., Liu, M. H. A Traceable E-Cash Transfer System Against Blackmail via Subliminal Channel. Electronic Commerce Research and Applications, 2009, 8(6), 327-333. https://doi.org/10.1016/j.elerap.2009.04.012

3. Chung, Y. F., Huang, K. H., Lee, H. H., Lai, F., Chen, T. S. Bidder-Anonymous English Auction Scheme with Privacy and Public Verifiability. Journal of Systems and Software, 2008, 81(1), 113-119. https://doi.org/10.1016/j. jss.2007.03.029

4. Fan, C. I., Wu, C. N., Sun, W. Z., Chen, W. K. Multi-Recastable E-Bidding Game with Dual-Blindness. Mathe-
ElGamal encryption are used to ensure transaction processing safety, and subliminal messages and blind signatures are used to ensure bidders' privacy and security. Moreover, a fair arbitration protocol was designed to ensure fair transactions.

Although the computation cost of the proposed scheme is higher than related works, the scheme is more secure and suitable for public engineering projects.

\section{Acknowledgements}

This research was supported by the Ministry of Science and Technology, Taiwan, R.O.C., under contract number MOST 106-2622-E-305-001-CC2, MOST 106-2221-E324 -013 and MOST103-2632-E-324-001-MY3. matical and Computer Modelling, 2013, 58(1-2), 68-78. https://doi.org/10.1016/j.mcm.2012.06.003

5. Harn, L., Gong, G. A Digital Signature with a Subliminal Channel. IEE Proceedings, Computers and Digital Techniques, 1997, 144(6), 387-389. https://doi. org/10.1049/ip-cdt:19971511

6. Hwang, M. S., Lu, E. J. L., Lin, I. C. Adding Timestamps to the Secure Electronic Auction Protocol. Data \& Knowledge Engineering, 2002, 40(2), 155-162. https:// doi.org/10.1016/S0169-023X(01)00048-9

7. Koblitz, N., Menezes, A. J., Vanstone, S. A. The State of Elliptic Curve Cryptography. Design, Codes and Cryptography, 2000, 19(2-3), 173-193. https://doi. org/10.1023/A:1008354106356 
8. Liaw, H. T., Juang, W. S., Lin, C. K. An Electronic Online Bidding Auction Protocol with Both Security and Efficiency. Applied Mathematics and Computation, 2006, 174(2), 1487-1497. https://doi.org/10.1016/j. amc.2005.06.016

9. Lin, D. R., Wang, C. I., Zhang, Z. K., Guan, D. J. A Digital Signature with Multiple Subliminal Channels and Its Applications. Computers \& Mathematics with Applications, 2010, 60(2), 276-284. https://doi.org/10.1016/j. camwa.2010.01.001

10. Lu, Y., Li, L., Peng, H., Yang, Y. A Biometrics and Smart Cards Based Authentication Scheme for Multi-Server Environments. Security and Communication Networks, 2015, 8(17), 3219-3228. https://doi.org/10.1002/ sec. 1246

11. Lu, Y.,Li,L., Yang, X., Yang, Y.Robust Biometrics Based Authentication and Key Agreement Scheme for Multi-Server Environments Using Smart Cards. PLoS ONE, 2015, 10(5), e0126323.doi:10.1371/journal.pone.0126323. https: //doi.org/10.1371/journal.pone.0126323

12. Menezes, A. J., Oorschot, P. C. V., Vanstone, S. A. Handbook of Applied Cryptography. CRC Press LLC, Boca Raton, 1996. https://doi.org/10.1201/9781439821916

13. Scott, M. Implementing Cryptographic Pairings. Proceedings of the 1st International Conference on Pairing-Based Cryptography, 2007, 177-196.
14. Seo, S. H., Choi, K. Y., Hwang, J. Y., Kim, S. Efficient Certificateless Proxy Signature Scheme with Provable Security. Information Sciences, 2012, 188(1), 322-337. https://doi.org/10.1016/j.ins.2011.11.005

15. Simmons, G. J. The Prisoner's Problem and the Subliminal Channel. Proceedings of Crypto '83, New York, 1983, 51-6r.

16. Simmons, G. J. The Subliminal Channel and Digital Signatures. Lecture Notes in Computer Science (LNCS), 1985, 209, 364-378. https://doi.org/10.1007/3-540-3975r-4_25

17. Simmons, G. J. Subliminal Communication Is Easy Using the DSA. Lecture Notes in Computer Science (LNCS), 1994, 765, 218-232. https://doi.org/10.1007/3540-48285-7_-18

18. Subramanian, S. Design and Verification of a Secure Electronic Auction Protocol. Proceedings of 17th IEEE Symposium on Reliable Distributed Systems, 1998, 204-210. https://doi.org/10.1109/RELDIS.1998.740497

19. Xiong, H., Chen, Z., Li, F. Bidder-Anonymous English Auction Protocol Based on Revocable Ring Signature. Expert Systems with Applications, 2012, 39(8), 70627066. https://doi.org/10.1016/j.eswa.2012.01.040

20. Zhang, Y., Liu, W., Lou, W., Fang, Y. Securing Mobile Ad Hoc Networks with Certificateless Public Keys. IEEE Transactions on Dependable and Secure Computing, 2006, 3(4), 386-399. https://doi.org/10.1109/TDSC.2006.58

\section{Summary / Santrauka}

Due to the rapid development of the Internet, many Internet applications have recently become very widely used. Internet security has therefore become an important issue. This paper proposes an electronic public engineering project bidding protocol via a subliminal channel. In the proposed scheme, the subliminal channel can protect a bidder's interests, while allowing an official agent to make a fair arbitration. The proposed scheme is non-repudiable, untraceable and offers fair arbitration of public engineering projects, but is also resistant to replay, forgery and insider attacks, thus enhancing both security and fairness.

Dèl sparčios interneto plètros daugelis interneto programų tapo labai plačiai naudojamos. Tai lèmè, kad interneto saugumas tapo svarbiu klausimu. Straipsnyje pateikiamas elektroninių viešujų inžinerinių projektų siūlymo per užslaptintą kanalą protokolas. Siūlomoje schemoje užslaptintas kanalas gali apsaugoti konkurso dalyvio interesus, tuo tarpu leidžiant oficialiam agentui atlikti sąžiningą arbitražą. Siūloma schema yra ne tik neatmetama, nesusekama ir siūlanti sąžiningą viešujų inžinerinių projektų arbitražą, bet taip pat ir atspari pakartojimo atakoms, klastojimui ir atakuotojams tinklo viduje. Straipsnio autoriu siūloma schema taip pat padidina tiek saugumą, tiek sąžiningumą. 